\title{
Impact of Propagation Model on Capacity in Small-cell Networks
}

\author{
Sofia Sousa ${ }^{1}$, Fernando J. Velez ${ }^{1,2}$ \\ ${ }^{1}$ Instituto das Telecomunicações -DEM \\ Universidade da Beira Interior, Faculdade de Engenharia \\ 6201-001 Covilhã, Portugal \\ fjv@ubi.pt, sofia.sousa@1x.it.pt
}

\author{
Jon M. Peha ${ }^{2}$ \\ ${ }^{2}$ Dept. of Electrical and Computer Engineering \\ Dept. of Engineering \& Public Policy \\ Carnegie Mellon University \\ Pittsburgh, PA 15213-3890, USA \\ peha@cmu.edu
}

\begin{abstract}
This work evaluates the impact of different path loss models on capacity of small cell (SC) networks, including the relationship between cell size and capacity. We compare four urban path loss models: the urban/vehicular and pedestrian test environment from the ITU-R M. 1255 Report, and the two-slope Micro Urban Line-of-Sight (LoS) and Non-Line-of-Sight (NLoS) models from the ITU-R 2135 Report. We show that when using the ITU-R two-slope model that considers the existence of a breakpoint in the behaviour of path loss, for coverage distances, $R$, up to break-point distance divided by reuse factor, supported cell throughput, $\boldsymbol{R}_{b \text {-sup }}$, is much lower than expected when traditional single-slope models are assumed. For $R$ s longer than $d_{B P} / r_{c c}$ the results for $\boldsymbol{R}_{b \text {-sup }}$ increase with $R$, whereas they are steady or decrease with $R$ when using the traditional single-slope propagation models. We conclude that the two-slope propagation model yields a significantly lower throughput per square $\mathrm{km}$ than a traditional one-slope model if and only if cell radius is small.
\end{abstract}

Keywords- LTE-Advanced, Carrier Aggregation, CNIR, ITU-R propagation model, Radio and network optimization, System capacity, Spectrum sharing, HetNet.

\section{I.INTRODUCTION}

In recent years, several propagation path loss models have been developed and proposed for cellular systems operating in different environments (outdoor, urban, suburban, rural, and indoor). The path loss model represents the reduction of the signal when it is propagating from the transmitter to the receiver, e.g., between the base station and mobile user. There are three different ways to model the path loss, as follows: deterministic, stochastic and empirical [1]. The deterministic model considers a specific transmitter location, a receiver location, and the properties of the environment. This type of model considers the electromagnetic wave propagation and requires a 3-D map of the propagation environment. In many cases, it is not possible to consider such a specific environment, and the appropriate approach is to consider channels that model the "typical", "worst case" or "best case" [2]. One example of the deterministic model is a ray-tracing model. The stochastic models represent the environment as a series of random variables, therefore requiring less information about the environment and the use of less processing power. An empirical model is based on observation and measurements. The classification of empirical models can be further divided into time dispersive and non-time dispersive. Time dispersive provides information about time dispersive characteristics of the channel, i.e., the multipath delay spread of the channel. Non-time dispersive consider various parameters, such as distance, antenna heights, frequency and transmitter power to predict average path loss.

The aim of cellular wireless network design is to optimize system capacity. In this work, we focus on evaluating the impact of considering different propagation models in the dimensioning process to understand the network coverage areas, the cochannel interference and the underlying system capacity. The more accurate/realistic propagation models is the more efficient the determination of the trade-off between cell size and capacity becomes. In the process of designing a mobile cellular network to meet given capacity requirement, it is essential to figure out how many small cells are needed. As choosing an adequate propagation model improves the optimization procedure, we compare the ITU-R 2135 model [3], applied to the Urban micro scenario, Line-of-Sight or Non-Line-of-Sight, and the Urban/Vehicular and Pedestrian model, defined in the ITU-R M.1255 Report [4] applied to small cells, operating at $2.6 \mathrm{GHz}$. This paper is an extended version from [5]. Figure 1 shows a general classification taxonomy that includes the different ways to model path loss.

The rest of the paper is organized as follows. Section II gives an overview of the considered propagation models and their application to the analysis of the frequency reuse trade-off. Section III compares the achievable supported cell physical throughput for different reuse patterns and propagation models. Section IV discusses the main lesson learned. Finally, conclusions are drawn in section $\mathrm{V}$.

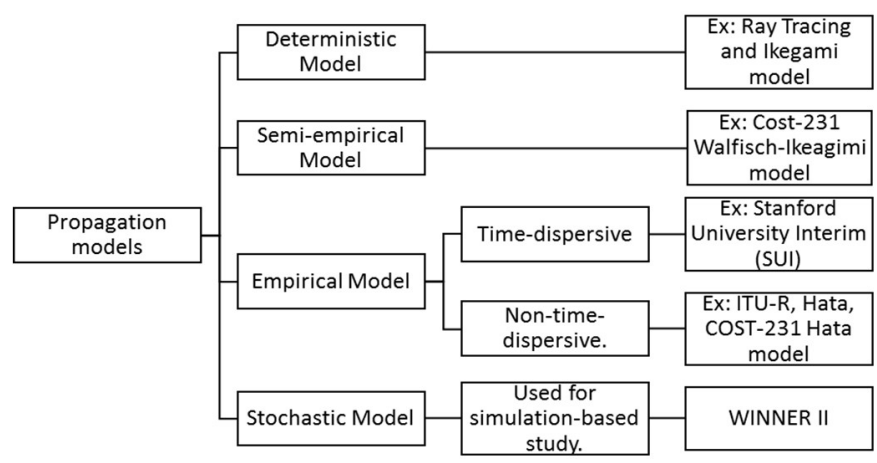

Figure 1 - General classification of the path loss models. 


\section{OVERVIEW OF THE PROPAGATION MODELS}

ITU-R was responsible for defining a global standard for the fourth generation of mobile communication systems known as international mobile telecommunications (IMT) - Advanced [6]. The ITU-R M 1225 Report has provided guidelines for evaluating a number of test environments. The scenarios under study are the outdoor-to-indoor/pedestrian test environments and vehicular test environment. Outdoor-to-indoor and pedestrian test environments is characterized by small cells and low transmitter power. Base stations with low antenna heights are located outdoors; pedestrian users are located on streets and inside buildings and residences. In turn, vehicular test environment is characterized by larger cells and higher transmitter power. The path loss model defined by ITU-R M.2135-1 Report proposes models that represent the channel behaviour through deterministic category. The deterministic category encompasses all models that describe the propagation channel for a specific transmitter location, receiver location and location. For evaluation of the IMT-Advanced candidates, the ITU-R WP D defines several test environments [3]. Each environment is characterized with certain user mobility, path loss characteristics and channel models, as well as system configuration parameters. The scenario evaluated in this work considers the microcellular scenario. The microcellular test environment focuses on small cells and high user densities and traffic loads in city centres and dense urban areas. The key characteristics of this test environment are high traffic loads, outdoor and outdoor-to-indoor coverage. The channel model for urban macro-cell scenario is called urban micro (UMi).

\section{A. Characterization of the Propagation Models}

The propagation characteristics for the outdoor-to-indoor/ pedestrian test environment are characterized by the following path loss model:

$P_{\text {Ledestrian }}=40 \log _{10}(d[\mathrm{~km}])+30 \cdot \log 10(f[\mathrm{MHz}])+49$

where $d$ is the separation between the mobile station and base station, in $\mathrm{km}$, and $f$ is the carrier frequency, in GHz. For $f=2.6$ $\mathrm{GHz}$ one obtains:

$P_{\text {L pedestrian }}(f=2.6 \mathrm{GHz})=40 \log 10\left(d_{[\mathrm{km}]}\right)+151.4492$

The propagation phenomena for vehicular/urban test environment is characterized by the following path loss model:

$P_{L \text { Urban }}=40\left(1-4 \times 10^{-3} h_{B S[\mathrm{~m}]}\right) \log _{10}\left(d_{[\mathrm{km}]}\right)-18 \log 10\left(h_{B S[\mathrm{~m}]}\right)+21$ $\log 10\left(f_{[\mathrm{MHz}]}\right)+80$

By considering the same assumptions as the previous model, $f=2.6 \mathrm{GHz}$ and $\Delta h_{b}=10 \mathrm{~m}$, the path loss, in $\mathrm{dB}$, is given by:

$P_{L \text { Urban }}(f=2.6 \mathrm{GHz})=38.40 \log 10\left(d_{[\mathrm{km}]}\right)+133.71$

where $\Delta h_{b}$ is the base station antenna height (in $\mathrm{m}$ ), measured from the average rooftop level. The UMi scenario is characterized by the following path loss model.

For the Outdoor scenario, there is a two-slope characteristic:

$P_{\text {L UMi LoS }}=22 \cdot \log _{10}\left(d_{[\mathrm{m}]}\right)+28.0+20 \log _{10}\left(f_{c[\mathrm{~Hz}]}\right)$, for $d<d_{B P}(5)$

$P_{L \text { UMi LoS }}=40 \cdot \log _{10}\left(d_{[\mathrm{m}]}\right)+7.8-18 \log _{10}\left(h_{B S}^{\prime}\right)-18 \cdot \log _{10}\left(h_{U T}^{\prime}\right)+$ $2 \log _{10}\left(f_{c[\mathrm{~Hz}]}\right)$, for $d>d_{B P}$
$P_{\text {L UMi NLoS }}=36.7 \cdot \log _{10}(d)+22.7+26 \cdot \log _{10}\left(f_{c}\right)$

where $h_{B S}=10 \mathrm{~m}$ and the considered street width is $20 \mathrm{~m}$, while the average building height is $20 \mathrm{~m}$. Variables $h_{B S[\mathrm{~m}]}^{\prime}=h_{B S}-1$ and $h_{U T[\mathrm{~m}]}^{\prime}=h_{U T}-1$ also stand. The break-point distance $d_{B P}$ is calculated by:

$d_{B P}=4 \cdot h^{\prime}{ }_{B S} \cdot h^{\prime}{ }_{U T} \cdot f_{c} / \mathrm{c}$

where $f_{\mathrm{c}}$ is the centre frequency, in Hertz, $c=3.0 \times 10^{8} \mathrm{~m} / \mathrm{s}$ is the propagation velocity in free space. Therefore, one obtains $d_{B P}$ UMi LoS $=156 \mathrm{~m}$.

By considering these assumptions, the path loss, in $\mathrm{dB}$, is given by:

$P_{\text {L UMi Los }}(d)=22 \cdot \log _{10}\left(d_{[\mathrm{m}]}\right)+36.29947$, for $d<156 \mathrm{~m}$

$P_{\text {L UMi LoS }}(d)=40 \cdot \log _{10}\left(d_{[\mathrm{m}]}\right)-3.12788$, for $d \geq 156 \mathrm{~m}$

$P_{L \text { UMi NLoS }}(d)=36.7 \cdot \log _{10}\left(d_{[\mathrm{m}]}\right)+33.48$,

The noise power at the receiver is calculated by:

$N[\mathrm{dBm}]=-174+10 \log 10 B W_{[\mathrm{Hz}]}+N_{f[\mathrm{~dB}]}$

where $B W$ is the bandwidth of the radio channel being used and $N_{f}$ is the noise figure at the receiver.

The assumed transmitter power and gains are $P_{t}=-7 \mathrm{dBW}$, $G_{t}=17 \mathrm{dBi}, G_{r}=0 \mathrm{dBi}, B W=10 \mathrm{MHz}, N_{f}=5 \mathrm{~dB}$ and $f=2.6 \mathrm{GHz}$.

\section{B. Cellular System}

In this section we describe a cellular system, and how to make coverage planning and frequency allocation, in terms of how to calculate the interference, carrier-to- interference ratio $(C / I)$ and carrier-to-interference-plus-noise ratio $(C N I R)$ in an OFDM system with static allocation scheme or fixed channel allocation. We consider a symmetrical hexagonal cell plan, a modulation scheme requiring a minimum CNIR and the transmitters use a constant transmitter power, $P_{t}$. Coverage planning is necessary to give mobile users a guarantee of the quality of the received signal for both the downlink (DL) and uplink (UL). One of the objectives is to design a wireless network where, for a given available bandwidth, the system achieves the highest capacity possible. We address the downlink of the system for the worst-case situation, where the UE is at the cell edge, where the channel is used in all these co-channel cells.

In a fully symmetrical hexagonal plan with a given frequency reuse pattern $K$, we consider the reuse distance, $D$, is $D=\sqrt{3 k} R$, where $R$ is the radius of the hexagonal cell. The possible values for $K$ are $K=1,3,4,7$, where $K=1$ is the case where all channels are used in all cells. As, for the very short coverage distances associated with small cells, the approximate $C / I$ formulation considered in the previous research work [7] has shown to be inadequate, a more detailed approach is sought in this work.

The $C / I$ ratio formulation used in the previous work is given by the following equation:

$$
\frac{C}{I}=\frac{1}{2\left(r_{c c}+1\right)^{-\gamma}+2 r_{c c}{ }^{-\gamma}+2\left(r_{c c}-1\right)^{-\gamma}} \approx \frac{r_{c c}{ }^{\gamma}}{6}
$$

where $r_{c c}$ is the co-channel reuse factor, given by $r_{c c}=D / R$. 
Instead, we have obtained a more detailed equation that represents $C / I$ with exact values for all the reuse distances, from the eNBs of the first, second and third tiers of co-channel cells (interferers) to the UE. Considering the first three tiers of interferers is a valid approximation, since the interference obtained from the second and third tier is negligible. In these equations we consider the exact position of each interferer, in each tier of interferers, in opposition to the equations with approximate values for the reuse distances. With hexagonal cell topologies for the macro- and pico- cellular layers, in the DL, as shown in Figure 2, for $K=3, C / I$ is given by:
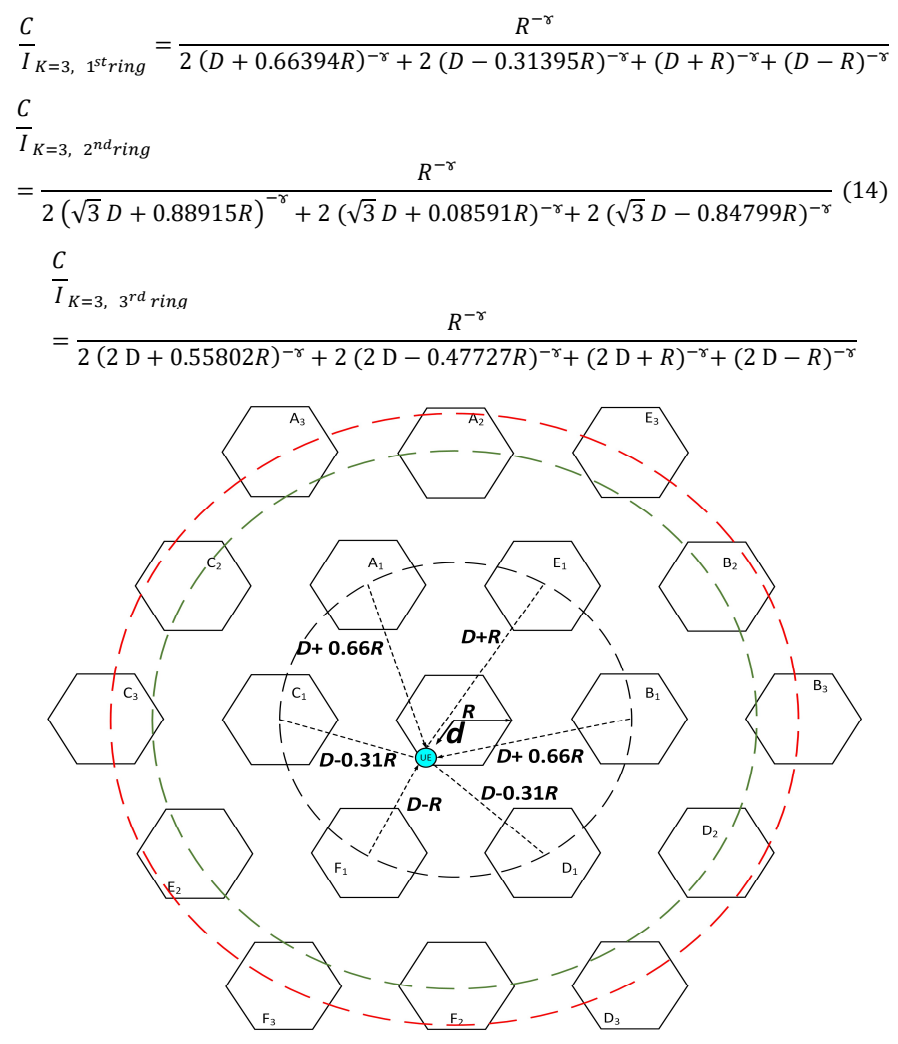

Figure 2 - Co-channel tiers (first, second and third tier) in the worst-case of hexagonal cellular systems in the downlink, for $K=3$.

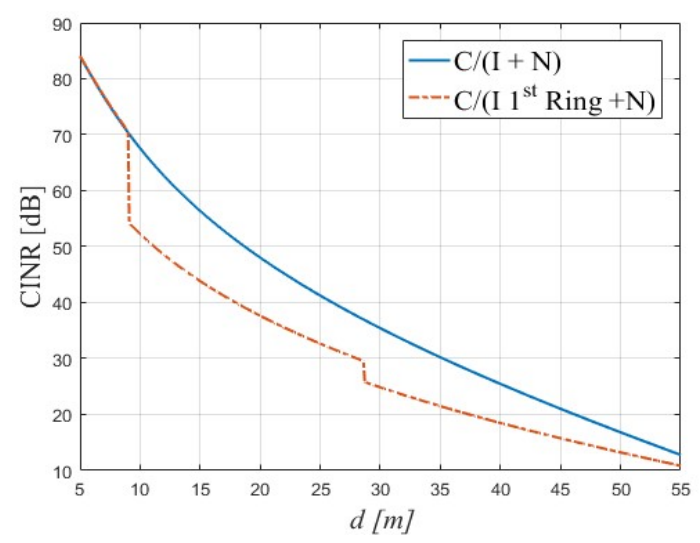

Figure 3 - Comparison of CNIR for the two-slope propagation model UMi LoS, $K=3$, between the approximate equation for $C /(I+N)$ and the equation, considering the exact distances from the eNBs interferers to the UE, for $\left(C /\left(I_{1 s t}\right.\right.$ Ring $+N$ ) both considering one tier of interference, for cells with $R=55 \mathrm{~m}$.
We have compared the values for the CNIR obtained with the formulation with exact reuse distances and the equation with approximate reuse distances. We consider short coverage distances inferior to $R_{o}$, where $R_{o}=d_{B P} / r_{c c}$. For $K=1 d_{B P}$ is $90 \mathrm{~m}$, for $K=3 d_{B P}$ is $52 \mathrm{~m}$ while, for $K=7, d_{B P}$ is $34 \mathrm{~m}$. For short coverage distances, and UMi LoS ITU-R 2135 model, $K=3$, for $R<d_{B P} / r_{c c}(=52 \mathrm{~m})$, the CNIR is slightly superior relatively to the formulation with exact reuse distances. For $52<R<77 \mathrm{~m}$, the CNIR obtained from this "exact" formulation is considerably lower (10-20 dB difference), as shown in Figure 3. From the results obtained for the CNIR for the single-slope propagation model, $K=3$, UMi NLoS and Pedestrian, we observe that CNIR is slightly superior $(\sim 2 \mathrm{~dB})$ for the formulation with exact distances from the three tiers of interference and slightly superior $(\sim 6 \mathrm{~dB})$, for the Urban case. The interference caused by the second and third rings is negligible. Figures 4 and 5 show how CNIR varies, with the distance $d$ from the cell centre to the UE within a cell, where $0 \leq d \leq R$, for cell coverage radii $R=30$ and $300 \mathrm{~m}$. The CNIRs obtained for UMi NLoS, Urban and Pedestrian are similar, as shown in Figure 4, since the respective propagation exponents are $\gamma=3.67,3.84$ and 4. However, in the UMi LoS scenario, for short coverage distances, as shown in Figure 4, since the propagation exponent is $\gamma=2.2$, the CNIR is considerably lower. For $R$ s longer than $R_{o}$, for UMi LoS, the obtained CNIR is gradually closer to the rest of the path loss curves, as for $R$ s longer than $d_{B P}$, since the propagation exponent of UMi LoS is $\gamma=4$, the obtained CNIR is higher than for the rest of the path loss models curves, as shown in Figure 5.
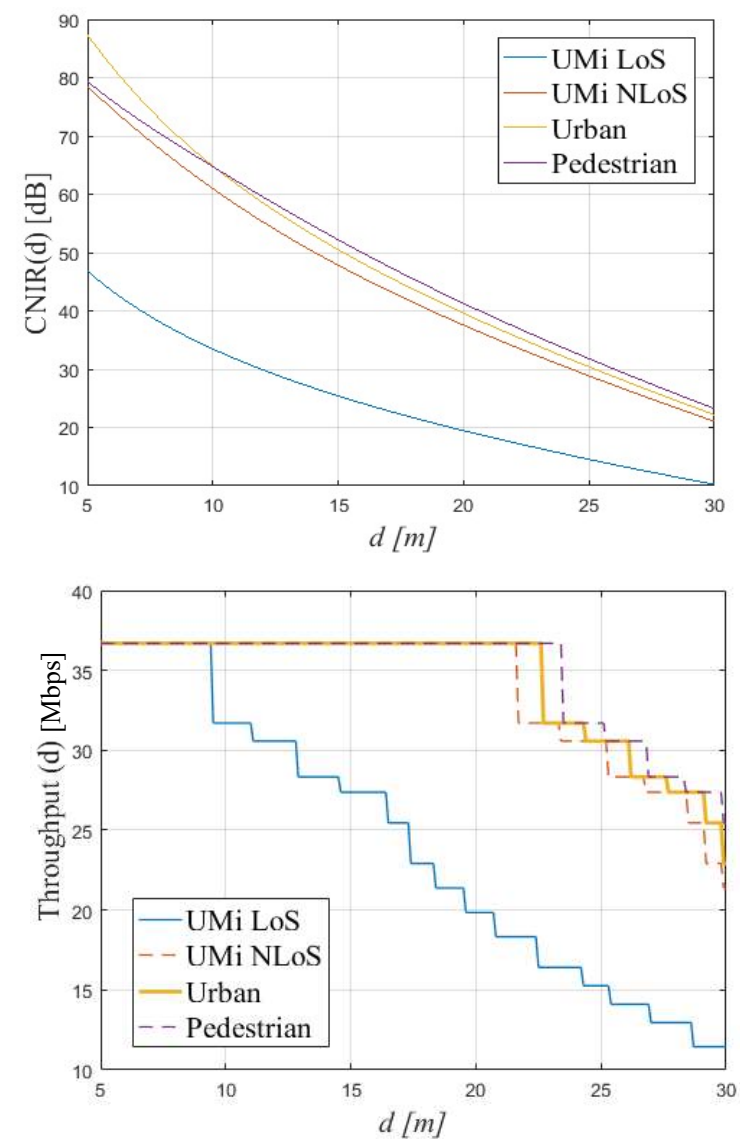

Figure 4 - Comparison of CNIR and throughput between the UMi LoS, UMi NLoS, Urban and Pedestrian propagation models, for $K=3$ and $R=30 \mathrm{~m}$. 

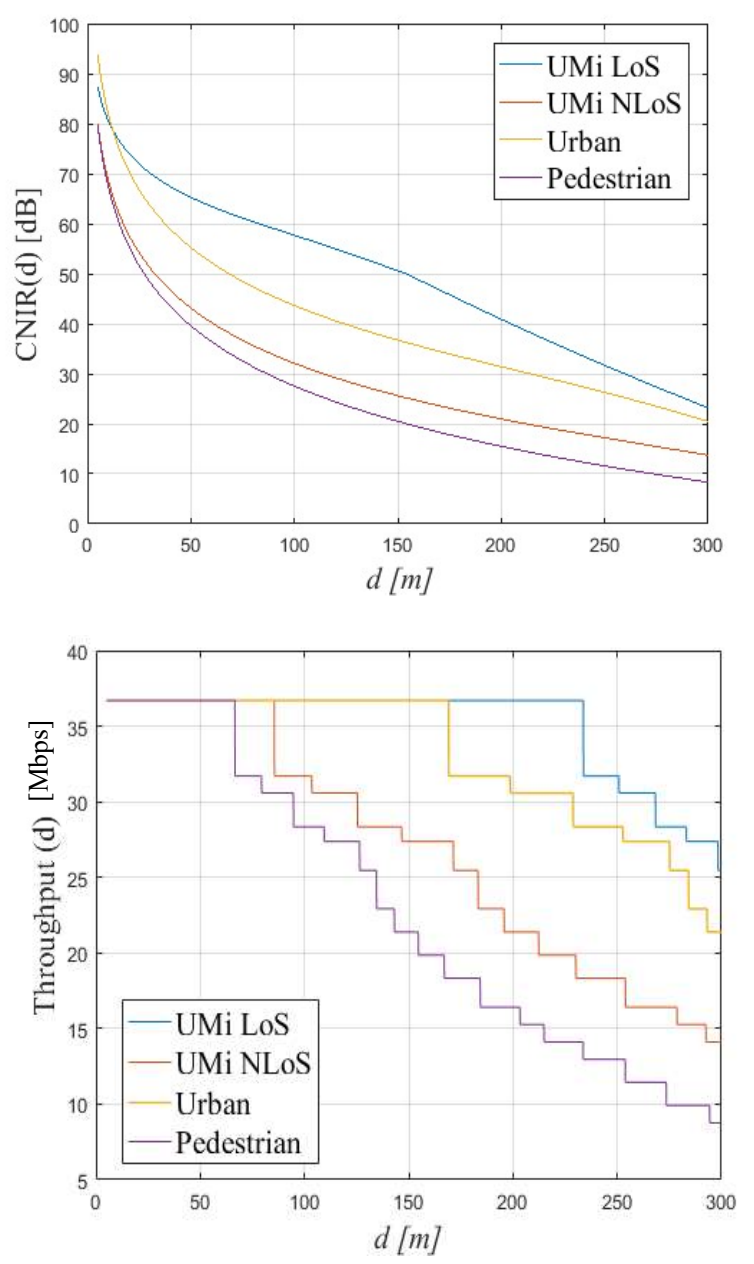

Figure 5 - Comparison of $C N I R$ and throughput between the UMi LoS, UMi NLoS, Urban and Pedestrian propagation models, for $K=3$ and $R=300 \mathrm{~m}$.

\section{Supported CELL/SEctor Physical ThroughPuT}

The analysis of LTE-A system capacity follows the formulation from [9] for an implicit function procedure to compute the supported cell physical throughput $\left(R_{b-s u p}\right)$. This analysis considers different values of the reuse pattern, e.g., $K=3$. To map $C N I R_{\text {min }}$ into the supported throughput, $R_{b}$, we have used the values for $C N I R_{\min }$ from [10]. By extrapolating the gathered information, it is possible to map the CNIR into MCS index, Modulation Order Transport Block Size (ITBS) index and TBS.

Figures 6, 7 and 8 show results for the supported throughput per cell, $R_{b \text {-sup }}$. Observing the supported throughput for cells with $R \mathrm{~s}$ shorter than $125 \mathrm{~m}$ (for $K=1$ ) and $R$ s shorter than $70 \mathrm{~m}$ (for $K=3$ ), more optimistic results are obtained with the Pedestrian path loss model, followed by the Urban, UMi NLoS and UMi LoS models. This means that the UMi LoS model presents the most pessimistic results for small cell coverage ranges. Nevertheless, for longer cell ranges, for example $R \geq 225 \mathrm{~m}(K=1), R \geq 150 \mathrm{~m}$ $(K=3)$ and $R \geq 160 \mathrm{~m}(K=7)$, the best results are obtained for UMi LoS, followed by Urban, UMi NLoS. The worst results are obtained for the Pedestrian Path Loss model. To understand the impact of considering a more realistic propagation model that considers the existence of a break-point distance in the behaviour of the path loss, in radio and network optimization, we analyse the supported throughput per unit area, $R_{b-u a}$, in Figures 9 to 12 . $R_{b-u a}$ is obtained by multiplying the number of cells per unit area by the supported throughput for $K=1,3$ and 7.

The reduction of the supported throughput while considering the UMi LoS propagation, $R_{b-u a}$ UMi LoS, is compared to the supported throughput while considering the Pedestrian propagation scenario, $R_{b-u a_{-} \text {Pedestrian }}$, is defined as $R_{e d} R_{b-u a}$ and is obtained by the ratio defined in the following equation:

$R e d \_R_{b-u a[\%]}=\frac{R_{b-u a_{-} P e d e s t r i a n}-R_{b-u a_{-} U M i_{L o S}}}{R_{b-u a \_P e d e s t r i a n}} \cdot 100$

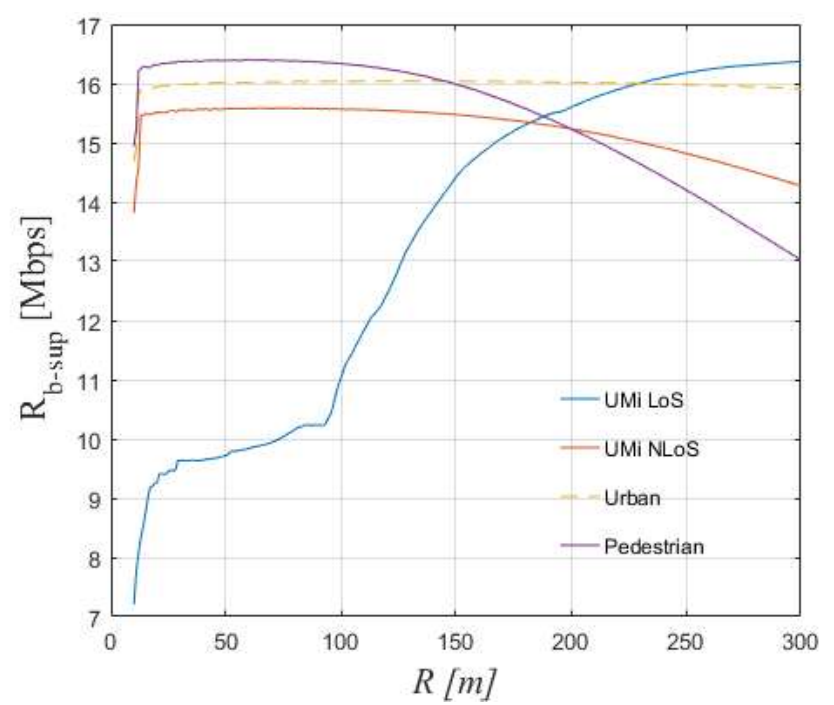

Figure 6 - Comparison of the equivalent supported throughput between the UMi LoS, UMi NLoS, Urban and Pedestrian path loss models, $K=1, B W=10$ $\mathrm{MHz}$.

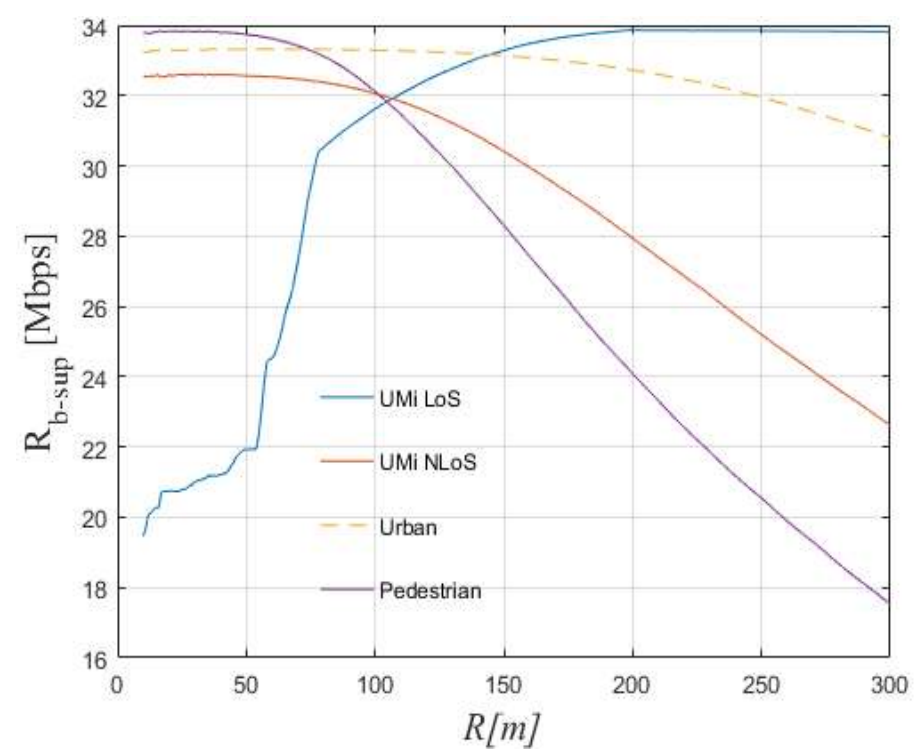

Figure 7 - Comparison of the equivalent supported throughput between the UMi LoS, UMi NLoS, Urban and Pedestrian path loss models, $K=3, B W=10$ $\mathrm{MHz}$. 


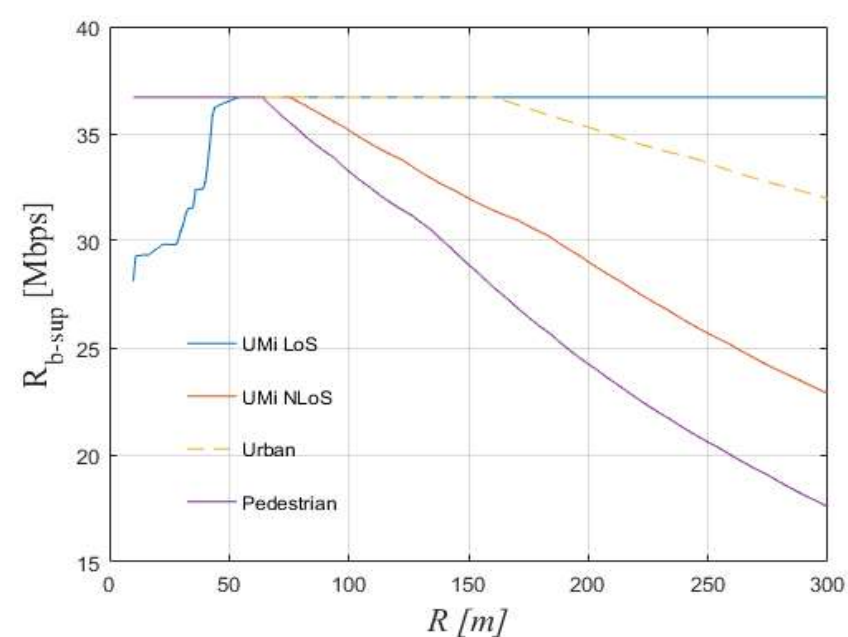

Figure 8 - Comparison of the equivalent supported throughput between the UMi LoS, UMi NLoS, Urban and Pedestrian path loss models, $K=7, B W=10$ $\mathrm{MHz}$.

For $K=1$, we observe in Figures 9 and 10 that, for cells with short coverage distances, for example, $R=40 \mathrm{~m}$, the supported throughput per unit area, $R_{b-u a}$, obtained with the two-slope model (UMi LoS) is reduced by $41.12 \%$ compared to the results that arise from applying the single slope model (Pedestrian scenario), as shown in Figure 10. For $K=3$ and 7, Figures 11 and 12 show that the two-slope model has reductions of $37.23 \%$ and $10.86 \%$ in $R_{b-u a}$ compared to the values obtained with the single slope model, respectively. Figure 10 shows the reduction of $R_{b-u a}$ for the two-slope model (UMi LoS) compared to $R_{b-u a}$ for the Pedestrian model, divided by $R_{b-u a_{-} P e d e s t r i a n}$, in $\%$. The results with the two-slope model exceeds the value obtained for $R_{b-u a}$ from the one-slope model for coverage distances longer than $R=180 \mathrm{~m}, R=96 \mathrm{~m}$ and $R=45$, for $K=1,3$ and 7, respectively. In fact, values of $R e d \_R_{b-u a}$ higher than zero mean a reduction of the throughput when considering the two-slope model, whereas negative values (obtained for $R \mathrm{~s}$ longer than these values) mean that the single-slope modes are more pessimistic in the determination of the supported throughput per unit area.

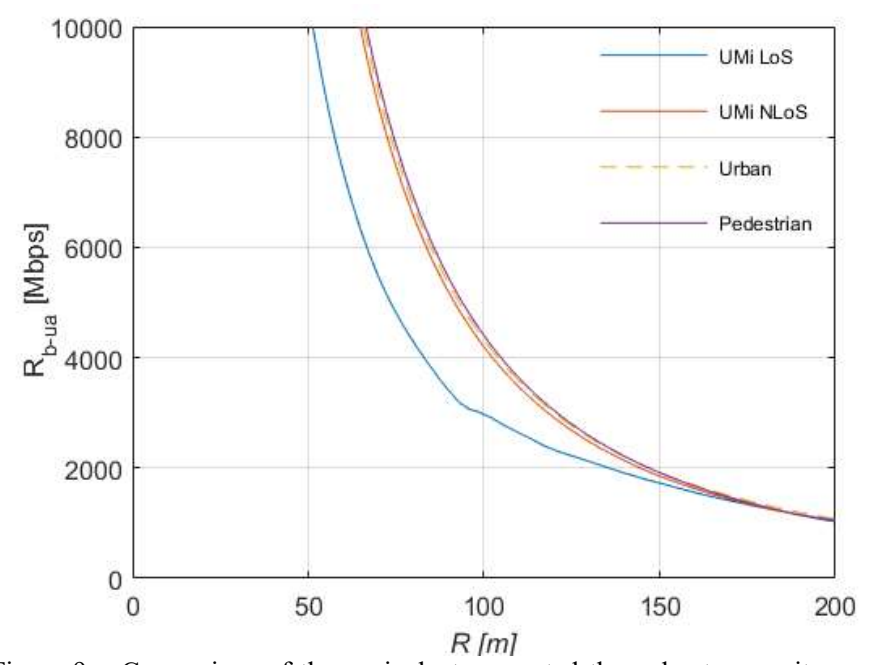

Figure 9 - Comparison of the equivalent supported throughput per unit area between the UMi LoS, UMi NLoS, Urban and Pedestrian path loss models, $K=1, B W=10 \mathrm{MHz}$.

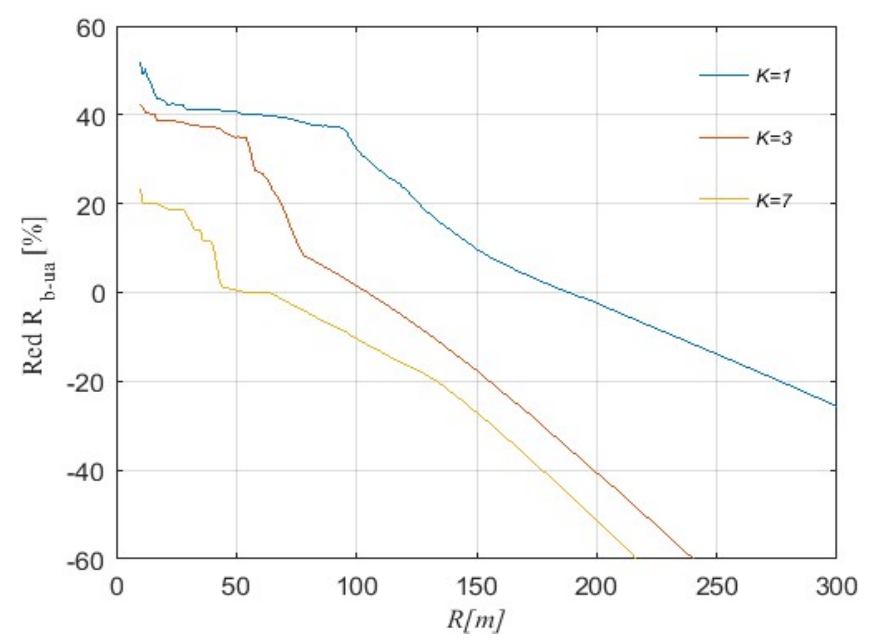

Figure 10 - Reduction of the equivalent $R_{b-u a}$ between the UMi LoS and Pedestrian path loss models in percentage, for $K=1,3$ and 7, $B W=10 \mathrm{MHz}$.

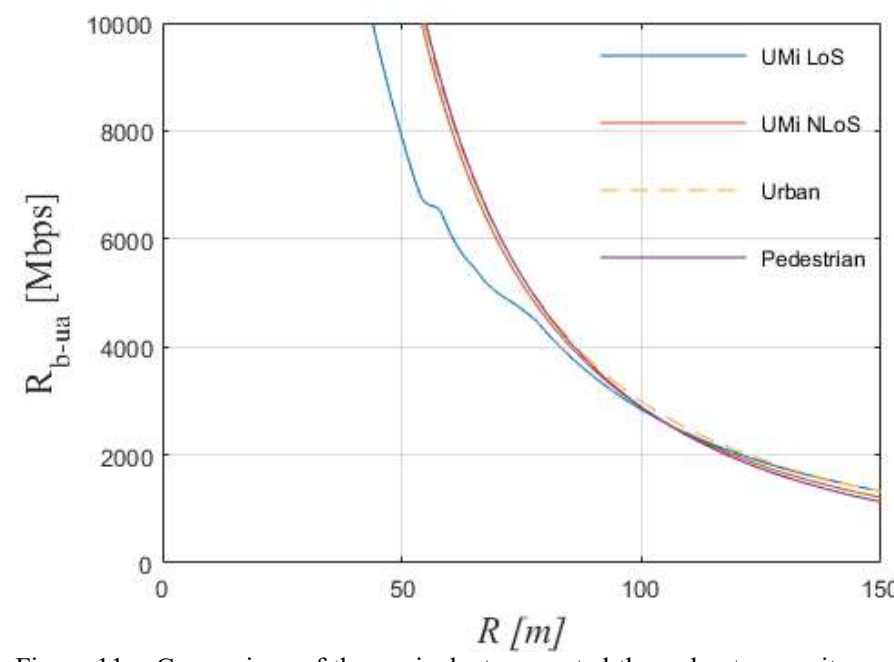

Figure 11 - Comparison of the equivalent supported throughput per unit area between the UMi LoS, UMi NLoS, Urban and Pedestrian path loss models, $K=3, B W=10 \mathrm{MHz}$.

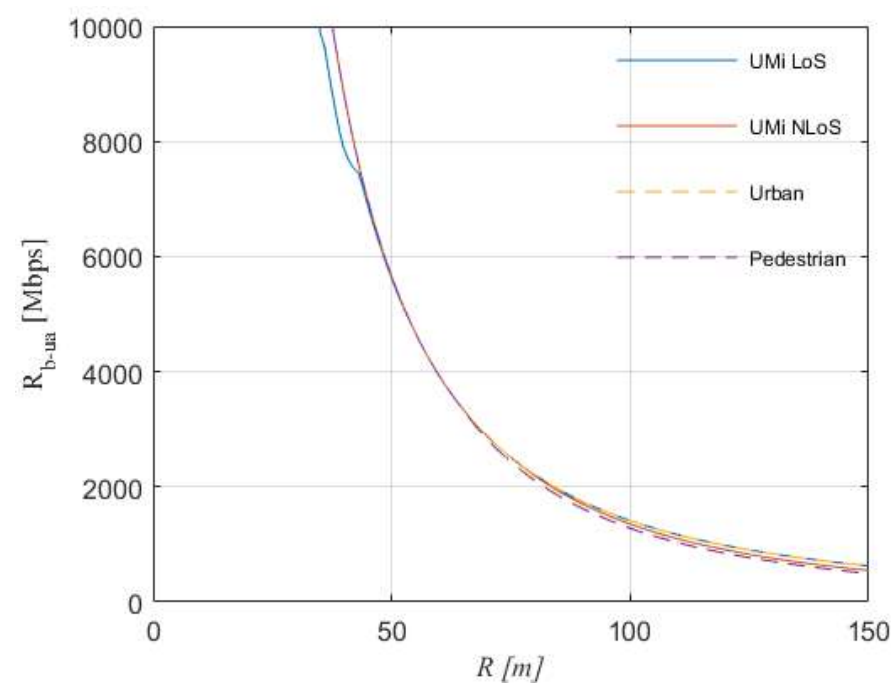

Figure 12 - Comparison of the equivalent supported throughput per unit area between the UMi LoS, UMi NLoS, Urban and Pedestrian path loss models, $K=7, B W=10 \mathrm{MHz}$. 
The two-slope model captures the actual behaviour of the propagation in small cell environments, whose break-point distance defines the change of the propagation characteristics. From this analysis, we conclude that, by considering the morerealistic ITU-R $2135 \mathrm{UMi}$ LoS propagation model, lower values of the throughput per unit area are achievable for shorter $R$ s while for longer $R$ s the consideration of the two-slope model leads to higher values of system capacity.

\section{LESSONS LEARNED}

To analyze the impact of different propagation models as well as having different propagation exponents for the UMi LoS (two-slope) propagation model, for different $R \mathrm{~s}$, we have drawn three dimensional (3D) graphs, where the behaviour of the PHY throughput $\left(R_{b}\right)$ mapped into MCS (with 29 levels, in the $z z$ axis) is represented, as shown in Figures 13-18. The cells size $(R)$ varies from $10 \mathrm{~m}$ to $300 \mathrm{~m}$. Because the range of distances $d$ is different while cell radii varies $(0 \leq d \leq R)$, in the view charts we have considered the normalized distance, defined as $d / R$, when representing the stepwise behaviour of the PHY throughput that defines the ring area which is using a certain MCS within the cell. The normalized distance represents the variation of $d$ from 0 to $R$. The cell radii is represented in the $y y$ axis while the normalized distance is shown in the $x x$ axis and varies from 0 to 1 .

There is a clear difference in the behaviour of $R_{b}$ between the two-slope and Pedestrain path loss models for $R$ s shorter than $R_{o}$. For the UMi LoS (two-slope) model, one can observe that a rapid decay occurs in the MCS (hence in the PHY throughput) for $R \mathrm{~s}$ shorter than $R_{0}$, due to the lowest propagation exponent (corresponding to extra co-channel interference). Clearly, for $R s$ longer that $R_{o}$, the behaviour changes and the decay is less pronounced, as generally speaking, for distances longer than $d_{B P} / r_{c c}$, the propagation exponent for the computation of the interference is higher.

It is wortwhile to analyze the impact of $C / I$ on the computation of $R_{b}$, hence in the computation of the supported throughput, through the implicit function formulation we are considering.

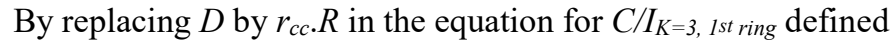
above, we can identify the distances $d_{0}$, in the numerator, and $d_{1}, d_{2}, d_{3}$, and $d_{4}$, in the denominator (which are raised to $-\gamma$ ). These break-point distances define the changes in behaviour of $R_{b-\text { sup }}$.

As an example, for $K=3$, by replacing $D$ by $3 \cdot R$ one obtains the following equation:

$\frac{C}{I}_{K=3,1^{\text {st }} \text { ring }}=\frac{R^{-\gamma}}{2(3.66394 \cdot R)^{-\gamma}+2(2.6861 \cdot R)^{-\gamma}+(4 \cdot R)^{-\gamma}+(2 \cdot R)^{-\gamma}}$

As such, $d_{0}=R, d_{1}=3.66394 \cdot R, d_{2}=2.6861 \cdot R, d_{3}=4 \cdot R, d_{4}=2 \cdot R$. If we equalize each of these distances to $d_{B P}$, we will obtain values of $R=156,42.58,58.08,39$ and $78 \mathrm{~m}$, respectively. Figure 17 represents $R_{b}$ for $K=3$ while considering the UMi LoS (twoslope) propagation model. The variation in behaviour around $R \mathrm{~s}$ equal to 40,60 and 80 and $156 \mathrm{~m}$ is very clear. They occur for different normalized distances and impact the throughput. In such partial "break-points", defined by the distances associated with interference $d_{1}, d_{2}, d_{3}$, and $d_{4}$, the propagation exponent changes from 2.2 to 4 , resulting in the decrease from interfeence beyond that distances. The improvement (increase) stops at $d_{0}=d_{B P}=156 \mathrm{~m}$, as the propagation exponent associated with coverage changes from to 2.2 to 4 ; hence, worse coverage occurs beyond this coverage range.

As for each $R, R_{b-\text { sup }}$ (Figures 6 to 8 ) is computed as an integral, which sums the contributions from the stepwise $R_{b}$ for all the distances, ranging from 0 to $R$, as established in [9], the area below the curve of $R_{b}$ has not got a sudden variation, and it atually has a continuous behaviour, as shown in Figure $7(K=3)$.

These 3D curves represent in a single view chart the joint contribution form different MCS, and facilitate the interpretation of the variation of $R_{b-s u p}$, as a measure of system capacity. They clearly show that, when cell radius is short, achievable throughput with a two-slope model is significantly lower, whereas for $R \mathrm{~s}$ longer than approximatelly $d_{B P}$, it tipically overcomes the values of $R_{b-\text { sup }}$ for the Pedestrian (single-slope) propagation model.

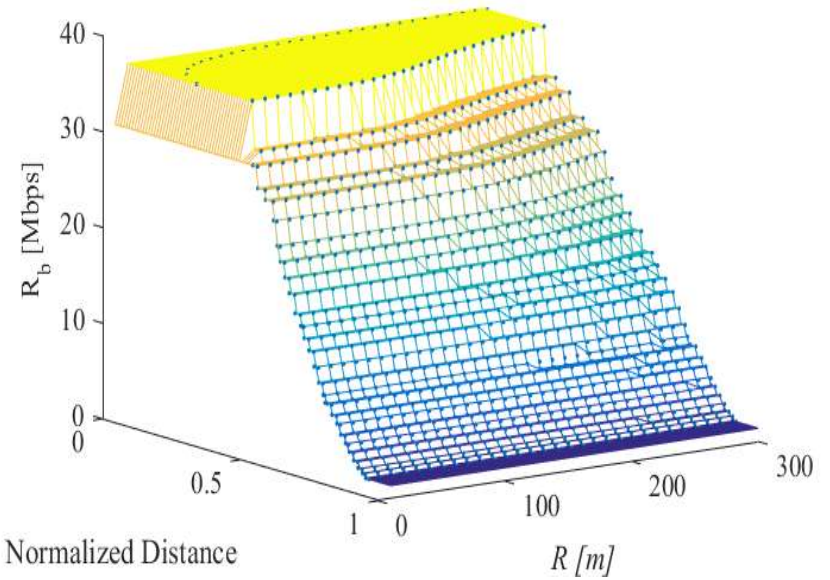

Figure $13-3 \mathrm{D}$ view graph for the supported throughput $\left(R_{b}\right)$ mapped into MCS (with 29 levels, in the $z z$ axis), obtained for $10 \leq R \leq 300 \mathrm{~m}$ for the Pedestrian propagation model and $K=1$.

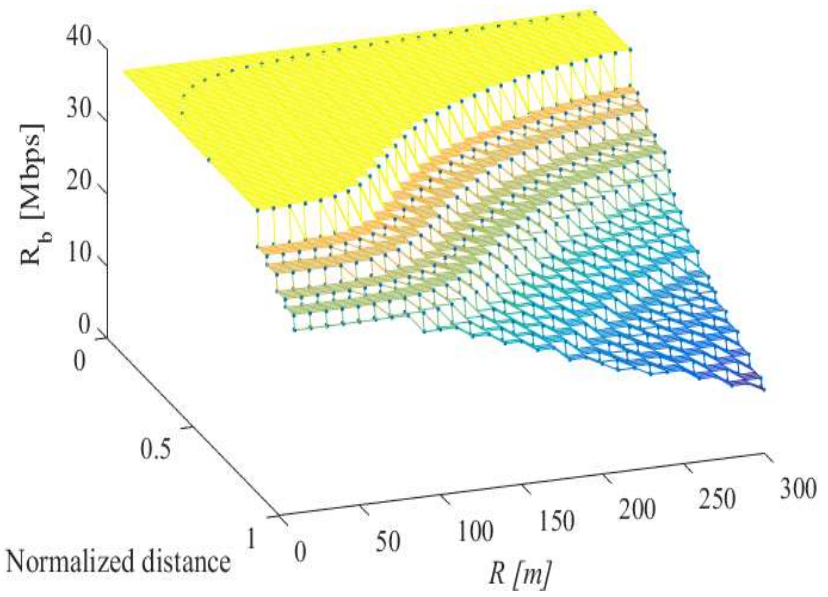

Figure $14-3 \mathrm{D}$ view graph for the supported throughput $\left(R_{b}\right)$ mapped into MCS (with 29 levels, in the $z z$ axis), obtained for $10 \leq R \leq 300 \mathrm{~m}$ for the Pedestrian propagation model and $K=3$. 


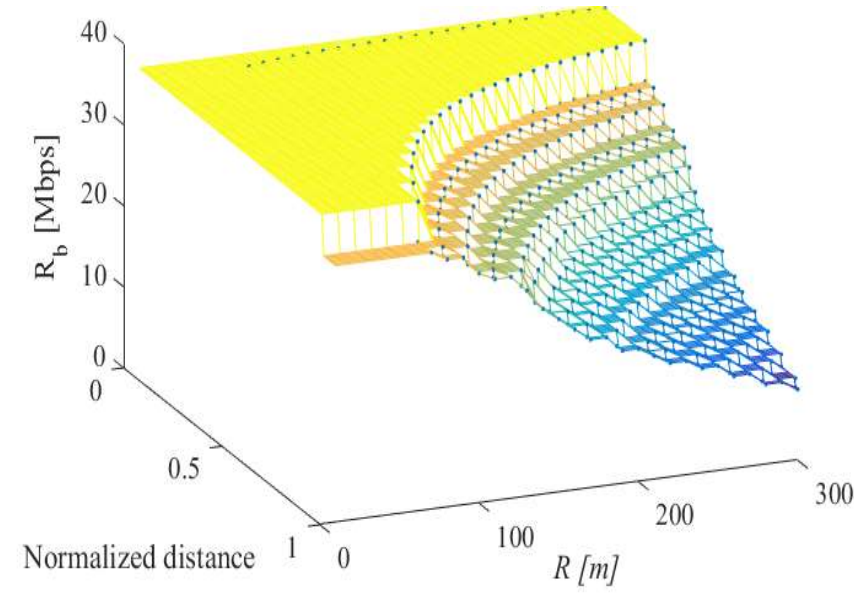

Figure $15-3 \mathrm{D}$ view graph for the supported throughput $\left(R_{b}\right)$ mapped into MCS (with 29 levels, in the $z z$ axis), obtained for $10 \leq R \leq 300 \mathrm{~m}$ for the Pedestrian propagation model and $K=7$.

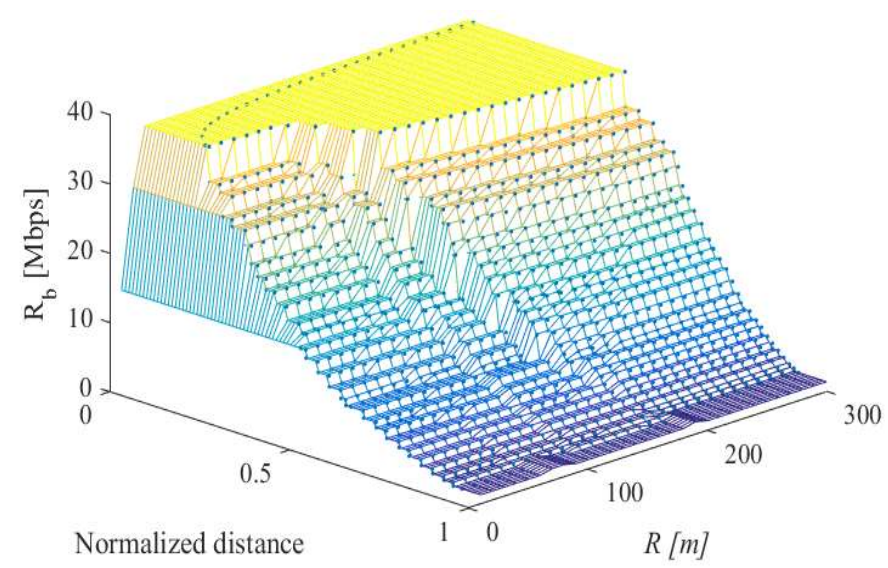

Figure $16-3 \mathrm{D}$ view graph for the supported throughput $\left(R_{b}\right)$ mapped into MCS (with 29 levels, in the $z z$ axis), obtained for $10 \leq R \leq 300 \mathrm{~m}$ for the UMi LoS propagation model and $K=1$.

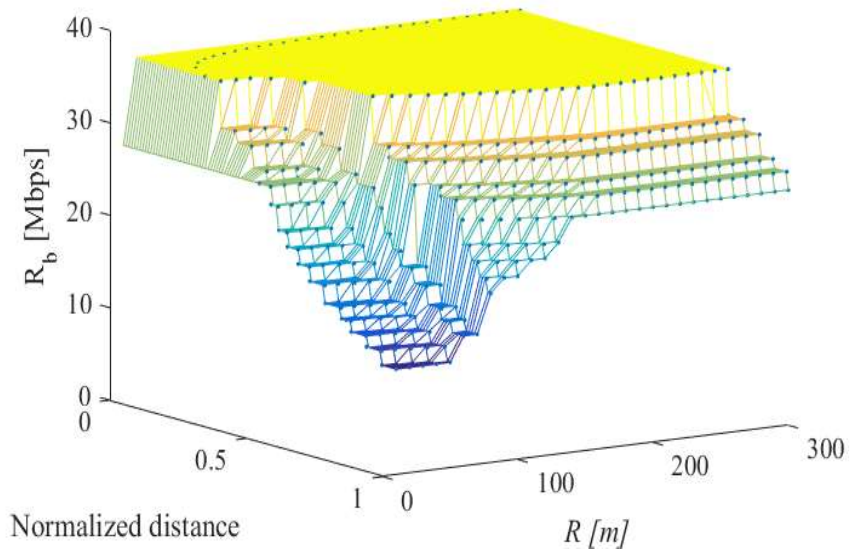

Figure $17-3 \mathrm{D}$ view graph for the supported throughput $\left(R_{b}\right)$ mapped into MCS (with 29 levels, in the $z z$ axis), obtained for $10 \leq R \leq 300 \mathrm{~m}$ for the UMi LoS propagation model and $K=3$.
Also, the reduction of the equivalent, $R_{b \text {-sup }}$, that shows that the gains in capacity per unit area from reducing cell size gets smaller, $20-45 \%$ lower, when $R s$ are shorter than $R_{0}$, calculated in Figure 11, is easily interpreted with a $3 \mathrm{D}$ curve.

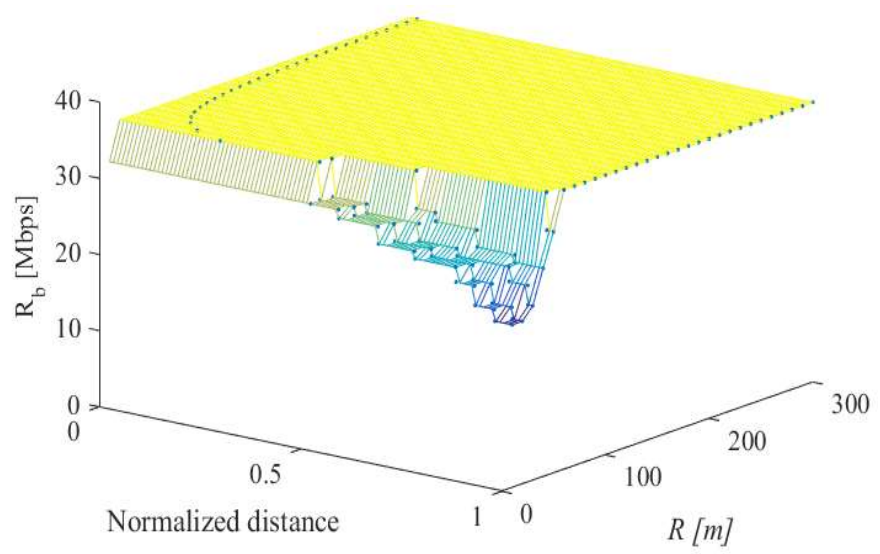

Figure $18-3 \mathrm{D}$ view graph for the supported throughput $\left(R_{b}\right)$ mapped into MCS (with 29 levels, in the $z z$ axis), obtained for $10 \leq R \leq 300 \mathrm{~m}$ for the UMi LoS propagation model and $K=7$.

\section{CONCLUSION}

This work has evaluated the impact of different path loss models on the system capacity of small cell networks. Also, we have obtained a more detailed equation that estimates the carrierto-interference ratio, $C / I$, with exact values for all the reuse distances from the eNBs of the first, second and third tiers of cochannel cells (interferers) to the UE. We have concluded that for short coverage distances, and for the Urban Micro Scenario Line-of-Sight (UMi LoS), ITU-R 2135, $K=3$, for $R<d_{B P} / r_{c c}$ (where $d_{B P}$ is the break-point distance and $r_{c c}$ is the reuse factor), the carrier-to-interference-noise-ratio (CNIR) is slightly superior if one considers the exact values for all reuse distances. For $K=3$ and $52 \mathrm{~m}<R<78 \mathrm{~m}$, the CNIR obtained by considering the exact values for all reuse distances for the three tiers of interferers is considerably lower (10-20 dB difference). The Urban model corresponds to the higher difference between the CNIR obtained with exact values for all reuse distances for the three tiers of interferers compared to the equations with approximate values for the reuse distances. When comparing the different path loss models, the CNIR for coverage distances shorter than $R_{o}=d_{B P} / r_{c c}$, where $R_{o}$ is $90 \mathrm{~m}$ for $K=1,52 \mathrm{~m}$ for $K=3$, and $34 \mathrm{~m}$ for $K=7$, for the UMi NLoS, Urban and Pedestrian scenarios is very similar, since the propagation exponents are $\gamma=3.67,3.84$ and 4, respectively, except for UMi LoS, since the propagation exponent is $\gamma=2.2$ (and therefore the CNIR is considerably lower). For $R$ s longer than $R_{o}$, the obtained CNIR for UMi LoS is gradually closer to the remaining path loss curves. For distances longer than $d_{B P}$, since the propagation exponent is $\gamma=4$ for UMi LoS, the obtained CNIR is higher than the rest of the path loss model curves, followed by the Urban path loss model, UMi NLoS and Pedestrian Path Loss Model. 
In summary, we learned from the analysis that by considering the realistic assumptions from the ITU-R two-slope model, for coverage distances, $R$, up to the break-point distance divided by $K, d_{B P} / r_{c c}, R_{b \text {-sup }}$ is much lower than expected when one assumes traditional single-slope models. For $R \mathrm{~s}$ longer than $d_{B P} / r_{c c}$ the results for $R_{b \text {-sup }}$ are increasing with $R$, whereas they are steady or decreasing with $R$ while considering the traditional singleslope propagation models. This increase is due to the existence of a low propagation exponent (slope) in term of coverage and a high slope in terms of interference for $d_{B P} / r_{c c} \leq R \leq d_{B P}$.

Recent research has found that a two-slope propagation model is more accurate than the traditional one-slope models [3]. We have found that these two models yield similar results if cell radius is large compared to the break-point of the two-slope model divided by reuse factor. However, when cell radius is small, throughput achievable with a two-slope model is significantly lower. We observed a throughput per area that is 20 to $45 \%$ lower in the scenarios considered. This difference in throughput exists because the one-slope model uses a higher propagation exponent for devices that are closer to the transmitter.

By analyzing the variation of the PHY throughput in detail, via $3 \mathrm{D}$ graphs, we learned that the changes in behaviour are justified by the values of $R$ corresponding to the points where the partial distances considered for the computation of interference achieve the break-point distance, $d_{B P}$. Consequently, when the cell radius is short, achievable throughput with a two-slope model is significantly lower, whereas for coverage distances longer than approximately $d_{B P}$, it typically overcomes the values of $R_{b-\text { sup }}$ for the Pedestrian (single-slope) propagation model.

Therefore, as cellular carriers reduce cell size to support growing traffic volume, use of traditional propagation models may produce designs with inadequate capacity. Moreover, assuming that the two-slope model is correct, these results also show that the gains in capacity per area from reducing cell size get smaller when cell radius falls below this $d_{B P} / r_{c c}$ threshold. This means that operators may find it more cost-effective at that point to meet their growing capacity needs by decreasing the frequency reuse factor or increasing spectrum holdings rather than decreasing cell size, assuming that spectrum is obtainable or that frequency reuse is not already at its minimum.

\section{ACKNOWLEDGMENT}

This work has been partially supported and funded by CREaTION, COST CA 15104, ECOOP, UID/EEA/50008/ 2013, EFATraS, SFRH/BSAB/113798/2015, 3221/BMOB/16 Carnegie Mellon University Portugal Faculty Exchange Programme grant, Bolsa BID/ICI-FE/Santander Universidades$\mathrm{UBI} / 2016$ and ORCIP.

\section{REFERENCES}

[1] V. S Abhayawardhana et al. "Comparison of empirical propagation path loss models for fixed wireless access systems," in Proc. of IEEE $61^{\text {st }}$ Vehicular Technology Conference. vol. 1. IEEE, 2005.

[2] IEEE $802.16 \mathrm{~m}-08 / 004 \mathrm{r} 5$, IEEE $802.16 \mathrm{~m}$ Evaluation Methodology Document, January 2009.

[3] Guidelines for evaluation of radio interface technologies for IMTAdvanced, Report ITU-R M.2135-1, Dec. 2009. (https://www.itu.int/dms pub/itu-r/opb/rep/R-REP-M.2135-1-2009PDF-E.pdf)

[4] Guidelines for evaluation of radio transmission technologies for IMT2000, Report ITU-R M.1255, 1997. (https://www.itu.int/dms pubrec/itur/rec/m/R-REC-M.1225-0-199702-I!!PDF-E.pdf)

[5] S. Sousa, F. J. Velez, J. M. Peha, "Impact of considering the ITU-R Two Slope Propagation Model in the System Capacity Trade-off for LTE-A HetNets with Small cells," in Proc. of $32^{\text {nd }}$ URSI GASS 2017, Montreal, Canada, Aug. 2017.

[6] LTE - Advanced A Practical Systems Approach to Understanding 3GPP LTE Releases 10 and 11 Radio Access Technologies, Academic Press, 2014.

[7] Fernando J. Velez, Sofia Sousa, Albena Mihovska and Ramjee Prasad, "Basic Limits for LTE-Advanced Radio and HetNet Optimization in the Outdoor-to-indoor Scenario", in Proc.of IEEE BlackSeaCom 2016, Varna, Bulgaria, June 2016.

[8] Daniel Robalo and F. J. Velez, "Economic trade-off in the optimization of carrier aggregation with enhanced multi-band scheduling in LTEAdvanced scenarios" EURASIP Journal on Wireless Communications and Networking, vol. 2015, no. 1, 2015, pp. 189 (doi: 10.1186/s13638015-0371-9).

[9] Fernando J. Velez, A. Hamid Aghvami and Oliver Holland, "Basic Limits for Fixed Worldwide Interoperability for Microwave Access Optimization Based in Economic Aspects," IET Communications Special Issue on WiMAX Integrated Communications, vol. 4, no. 9, June 2010, pp. 1116-1129 (available online DOI 10.1049/iet-com.2009.0190).

[10] 3GPP, TS 36.212, V11.3.0. Technical Specification Group Radio Access Network; Evolved Universal Terrestrial Radio Access (E-UTRA); Multiplexing and channel coding, 3GPP Std., June 2013. 